\title{
Siacci's Theorem According to Darboux Frame
}

\author{
Kahraman Esen Özen, Murat Tosun and Mahmut Akyig̃it
}

\begin{abstract}
The resolution of the acceleration vector of rigid body moving along a space curve is well known thanks to Siacci [1]. In this resolution, the acceleration vector is stated as the sum of two special oblique components in the osculating plane of the point of the curve. In this paper, we have studied the Siacci's theorem for the curves on regular surfaces in 3-dimensional Euclidean space. Also, an example is given for a helix lying on a cylinder.
\end{abstract}

\section{Introduction}

The theory of surfaces in 3-dimensional Euclidean space had already been developed widely when the Frenet-Serret formulas were found out by Serret and Frenet. The adaptation of the method of moving frames to the study of curves on surfaces was carried out by J. G. Darboux in the light of FrenetSerret approach to curves [2]. This natural moving frame which is constructed on a surface is called as Darboux frame [3]. The Darboux frame exists at all non-umbilic points of a surface. Therefore, it exists at all the points of a curve on a regular surface.

In kinematics, it is well known that a particle moving along a curve in 3dimensional Euclidean space has an acceleration which is obtained by differentiating velocity with respect to time parameter. To state the acceleration vector as the sum of its tangential and normal components, which are orthogonal to each other, is useful for numerous applications. However, to state the acceleration vector as the sum of its tangential and radial components, which

Key Words: Kinematics, Siacci's theorem, Darboux frame.

2010 Mathematics Subject Classification: Primary 53A05; Secondary 53A17.

Received: 14.12 .2016

Accepted: 31.01.2017 
do not need to be orthogonal to each other, is more useful for motions where the angular momentum of the particle is constant. The success of stating the acceleration in this form belongs to F. Siacci. In the Siacci's resolution of the acceleration vector, the tangent component lies along the tangent on the path while the radial component is directed from the particle towards the foot of the perpendicular that is from an arbitrary fixed origin to the instantaneous osculating plane to the path [4]. In the literature, there have been numerous studies about the Siacci's theorem. After F. Siacci, firstly, E. T. Whittaker dealt with Siacci's theorem and gave a geometrical proof of Siacci's theorem in the plane [5]. After that, N. Grossman gave a more modern proof in [6] (but the concomitant diagram was misdrawn). Recently, J. Casey has studied on the Siacci's theorem. According to J. Casey, although Siacci's formulas were quite striking, his expression of the theorem was not precise; also his proof was cumbersome. Therefore, J. Casey presented a proof of Siacci's theorem in the space which is based on the Frenet-Serret formulas [4]. Recently, M. Y. Yllmaz et al. [7] have studied Siacci's theorem for curves in Finsler Manifold $\mathbf{F}^{3}$.

In this paper, firstly, we have reviewed the Frenet-Serret frame and Darboux frame to disambiguate the ensuing sections. Afterwards, we have studied the Siacci's theorem for the curves on regular surfaces in $E^{3}$ by considering the statement and proof of Siacci's theorem inspired from [4]. Furthermore, we have given an example for circular helices.

\section{Preliminaries}

Let 3 -dimensional Euclidean space $E^{3}$ be provided with the standard scalar product

$$
\langle\mathbf{x}, \mathbf{y}\rangle=x_{1} y_{1}+x_{2} y_{2}+x_{3} y_{3}
$$

where $\mathbf{x}=\left(x_{1}, x_{2}, x_{3}\right)$ and $\mathbf{y}=\left(y_{1}, y_{2}, y_{3}\right)$ are arbitrary vectors in $E^{3}$. The norm of a vector $\mathbf{x}$ in $E^{3}$ is expressed by $\|\mathbf{x}\|=\sqrt{\langle\mathbf{x}, \mathbf{x}\rangle}$. A curve in $E^{3}$ $\alpha(s)$ is a unit speed curve if $\left\|\alpha^{\prime}(s)\right\|=1$ where $\alpha^{\prime}(s)$ is the tangent vector of $\alpha(s)$. In this case, $s$ is called arc-length parameter of the curve $\alpha(s)$.

The moving Serret-Ferent frame of the unit speed curve $\alpha(s)$ is denoted as $\{\mathbf{T}(s), \mathbf{N}(s), \mathbf{B}(s)\}$ which consist of a unit tangent vector $\mathbf{T}$, the unit principal normal vector $\mathbf{N}$ and the unit binormal vector $\mathbf{B}$. Then the derivative formulas of Frenet-Serret frame in the matrix form are given as

$$
\left(\begin{array}{l}
\mathbf{T}^{\prime} \\
\mathbf{N}^{\prime} \\
\mathbf{B}^{\prime}
\end{array}\right)=\left(\begin{array}{ccc}
0 & \kappa & 0 \\
-\kappa & 0 & \tau \\
0 & -\tau & 0
\end{array}\right)\left(\begin{array}{l}
\mathbf{T} \\
\mathbf{N} \\
\mathbf{B}
\end{array}\right)
$$


Here, the functions $\kappa(s)=\left\|\mathbf{T}^{\prime}(s)\right\|$ and $\tau(s)=-\left\langle\mathbf{B}^{\prime}(s), \mathbf{N}(s)\right\rangle$ are called the curvature and the torsion of the curve $\alpha(s)$, respectively [8]. Let $\alpha: I \subset R \rightarrow$ $M$ be a unit speed curve which lies on the regular surface $M$. Then there exists the Darboux frame denoted as $\{\mathbf{T}, \mathbf{Y}, \mathbf{U}\}$ along the curve $\alpha . \mathbf{T}$ is the unit tangent vector of $\alpha, \mathbf{U}$ is the unit normal vector of $M$ restricted to $\alpha$, and $\mathbf{Y}$ is the unit vector given as $\mathbf{Y}=\mathbf{U} \times \mathbf{T}$. Since $\mathbf{T}$ is mutual in both Darboux and Frenet-Serret frame, the other vectors of each frames lie in the same plane except $\mathbf{T}$. Therefore, the relation between this two frames could be given in the matrix form by

$$
\left(\begin{array}{c}
\mathbf{T} \\
\mathbf{Y} \\
\mathbf{U}
\end{array}\right)=\left(\begin{array}{ccc}
1 & 0 & 0 \\
0 & \cos \phi & -\sin \phi \\
0 & \sin \phi & \cos \phi
\end{array}\right)\left(\begin{array}{c}
\mathbf{T} \\
\mathbf{N} \\
\mathbf{B}
\end{array}\right),
$$

where $\phi$ is the angle between the vectors $\mathbf{Y}$ and $\mathbf{N}$ or likewise $\mathbf{U}$ and $\mathbf{B}$ $[9,10,11]$. Additionally, the derivative formulas of Darboux frame are given as

$$
\left(\begin{array}{l}
\mathbf{T}^{\prime} \\
\mathbf{Y}^{\prime} \\
\mathbf{U}^{\prime}
\end{array}\right)=\left(\begin{array}{ccc}
0 & k_{g} & k_{n} \\
-k_{g} & 0 & \tau_{g} \\
-k_{n} & -\tau_{g} & 0
\end{array}\right)\left(\begin{array}{l}
\mathbf{T} \\
\mathbf{Y} \\
\mathbf{U}
\end{array}\right)
$$

Here, the functions $k_{g}, k_{n}$, and $\tau_{g}$ are called geodesic curvature, normal curvature, and geodesic torsion of the curve $\alpha$, respectively [2]. With the help of (2) and (3), $k_{g}, k_{n}$, and $\tau_{g}$ satisfy the following equations:

$$
\begin{array}{ll}
k_{g}(s)=\kappa(s) \cos \phi, & \kappa^{2}(s)=k_{g}{ }^{2}(s)+k_{n}{ }^{2}(s), \\
k_{n}(s)=\kappa(s) \sin \phi, & \phi=\arctan \left(k_{n} / k_{g}\right), \\
\tau_{g}(s)=\tau(s)-\phi^{\prime}(s) . &
\end{array}
$$

Let $P$ be a particle whose mass is $m$ and which moves on a regular surface $M$, and let an arbitrary fixed origin $O$ be chosen in $E^{3}$. Also let $\mathbf{x}$ be the position vector of $P$ at time $t$ and $C$ be the oriented curve traced out by $P$ with the arc length parameter $s$ such that the arc length of $C$ corresponds to time $t$. Therefore, the unit tangent vector for the curve $C$ is given as

$$
\mathbf{T}=\mathbf{x}^{\prime}=\frac{d \mathbf{x}}{d s} .
$$

With the aid of (1) and (5), the velocity vector $\mathbf{v}$ and acceleration vector $\mathbf{a}$ of $P$ at time $t$ can be written as

$$
\mathbf{v}=\dot{\mathbf{x}}=v \mathbf{T}, v=\dot{s}, \quad \mathbf{a}=\dot{\mathbf{v}}=\dot{v} \mathbf{T}+\kappa v^{2} \mathbf{N},
$$

where $\frac{d}{d t}$ a superposed dot denotes differential with respect to $t$. Since $\mathbf{v}$ can be stated as a function of $s$ along $C$, the acceleration vector can be expressed 
as

$$
\mathbf{a}=v \frac{d v}{d s} \mathbf{T}+\kappa v^{2} \mathbf{N}
$$

and so a always lies in the osculating plane of $C$ at $P,[4]$.

Now, considering (3) and (5), the velocity vector $\mathbf{v}$ and acceleration vector $\mathbf{a}$ of $P$ at time $t$ with respect to Darboux basis are given as

$$
\begin{aligned}
& \mathbf{v}=\dot{\mathbf{x}}=v \mathbf{T} \\
& v=\dot{s} \\
& \mathbf{a}=\dot{\mathbf{v}}=\dot{v} \mathbf{T}+k_{g} v^{2} \mathbf{Y}+k_{n} v^{2} \mathbf{U}
\end{aligned}
$$

Likewise, this last equation can be written as

$$
\mathbf{a}=v \frac{d v}{d s} \mathbf{T}+k_{g} v^{2} \mathbf{Y}+k_{n} v^{2} \mathbf{U}
$$

From (4), we have the equations

$$
k_{g}=\sqrt{{k_{n}{ }^{2}+k_{g}^{2}}^{2}} \cos \phi \text { and } k_{n}=\sqrt{{k_{n}{ }^{2}+k_{g}{ }^{2}}^{2}} \sin \phi
$$

By applying these equations into (7), we obtain

$$
\mathbf{a}=v \frac{d v}{d s} \mathbf{T}+\sqrt{{k_{n}{ }^{2}+k_{g}{ }^{2}}^{2}(\cos \phi \mathbf{Y}+\sin \phi \mathbf{U})}
$$

Thus, the acceleration vector of $P$ lies in the plane $S_{\mathbf{p}}\{\mathbf{T}, \cos \phi \mathbf{Y}+\sin \phi \mathbf{U}\}$ of $C$ at $P$, invariably.

By considering $(6)_{1}$ and using the definition of angular momentum of $P$ about $O$, we obtain

$$
\mathbf{H}_{O}=\mathbf{x} \wedge m \mathbf{v}=\mathbf{x} \wedge m v \mathbf{T}
$$

and so it can be said that $\mathbf{H}_{O}$ lies in the plane perpendicular to vectors $\mathbf{T}$ and $\mathrm{x}$.

\section{Siacci's Theorem for the Curves on Regular Surfaces}

Since a particle, which moves along a curve, can be seen as a point of this curve, the aforesaid particle $P$ has a position vector in terms of the Darboux basis of the curve $C$. Let the position vector of $P$ on the Darboux basis be resolved as

$$
\mathbf{x}=\mathrm{q} \mathbf{T}-\mathrm{p}(\cos \phi \mathbf{Y}+\sin \phi \mathbf{U})+\mathrm{b}(-\sin \phi \mathbf{Y}+\cos \phi \mathbf{U})
$$


where

$$
\begin{aligned}
& \mathrm{q}=\langle\mathbf{x}, \mathbf{T}\rangle, \\
& \mathrm{p}=-\langle\mathbf{x}, \cos \phi \mathbf{Y}+\sin \phi \mathbf{U}\rangle, \\
& \mathrm{b}=\langle\mathbf{x},-\sin \phi \mathbf{Y}+\cos \phi \mathbf{U}\rangle .
\end{aligned}
$$

Let us denote a vector $\mathbf{r}$ in the plane $S_{\mathbf{p}}\{\mathbf{T}, \cos \phi \mathbf{Y}+\sin \phi \mathbf{U}\}$ as follows:

$$
\mathbf{r}=\mathrm{q} \mathbf{T}-\mathrm{p}(\cos \phi \mathbf{Y}+\sin \phi \mathbf{U}) .
$$

Then we get

$$
\mathrm{r}^{2}=\langle\mathbf{r}, \mathbf{r}\rangle=\mathrm{p}^{2}+\mathrm{q}^{2},
$$

where $r$ is the length of the vector $\mathbf{r}$ (Fig. 1).

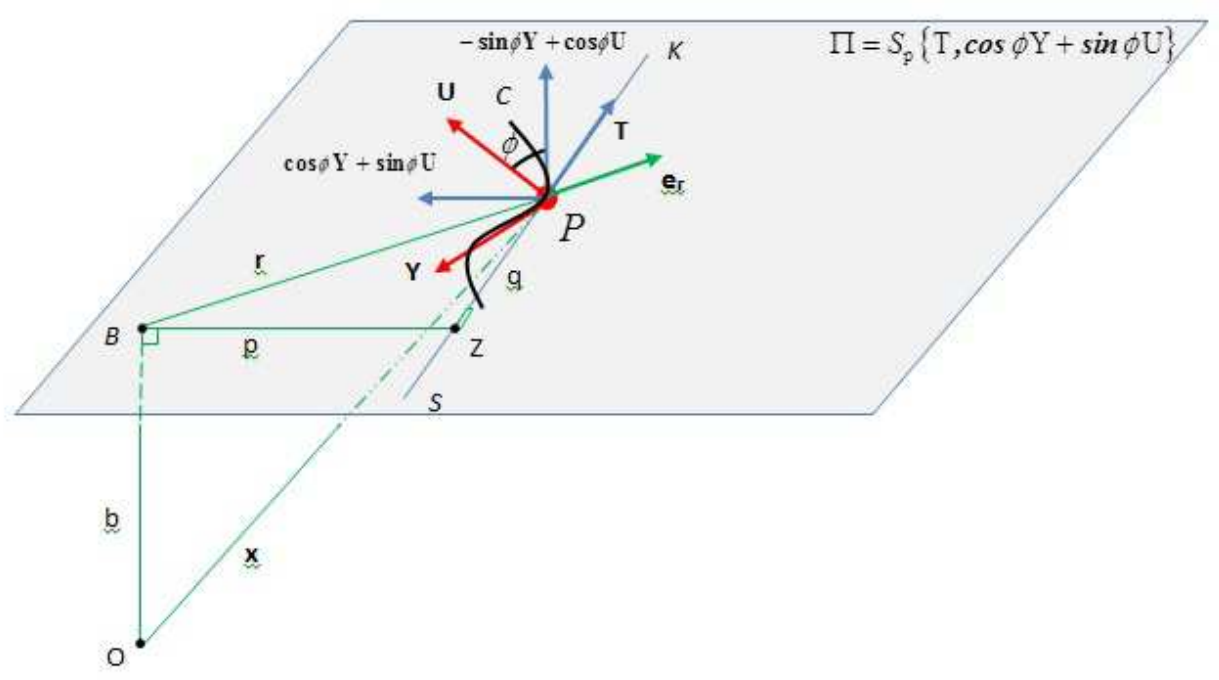

Figure 1: A particle $P$ moves along $C$, which is a curve on a regular surface in 3 -dimensional Euclidean space. The normal of plane $\Pi$ is $(-\sin \phi \mathbf{Y}+\cos \phi \mathbf{U})$. The tangent line to $C$ at $P$ is the line $d$. Also $B$ is the foot of the perpendicular from origin $O$ to the plane $\Pi$, and $B P$ is perpendicular to the tangent line. The vector $B P$ is shown with $\mathbf{r}$, and $\mathbf{e}_{\mathbf{r}}$ is the unit vector in direction of $B P$. 
By differentiating the equation (10) with respect to arc length parameter, we get

$$
\begin{aligned}
\mathbf{T}= & \left(\mathrm{q}^{\prime}+k_{g} \mathrm{~b} \sin \phi+k_{g} \mathrm{p} \cos \phi-k_{n} \mathrm{~b} \cos \phi+k_{n} \mathrm{p} \sin \phi\right) \mathbf{T} \\
& +\left(k_{g} \mathrm{q}-\mathrm{b}^{\prime} \sin \phi-\phi^{\prime} \cos \phi \mathrm{b}-\mathrm{p}^{\prime} \cos \phi+\phi^{\prime} \sin \phi \mathrm{p}-\tau_{g} \mathrm{~b} \cos \phi+\tau_{g} \mathrm{p} \sin \phi\right) \mathbf{Y} \\
& +\left(k_{n} \mathrm{q}+\mathrm{b}^{\prime} \cos \phi-\phi^{\prime} \sin \phi \mathrm{b}-\mathrm{p}^{\prime} \sin \phi-\phi^{\prime} \cos \phi \mathrm{p}-\tau_{g} \mathrm{~b} \sin \phi-\tau_{g} \mathrm{p} \cos \phi\right) \mathbf{U}
\end{aligned}
$$

Since the vectors $\mathbf{T}, \mathbf{Y}$, and $\mathbf{U}$ are linearly independent, the following equations hold:

$$
\begin{aligned}
& q^{\prime}+k_{g} \mathrm{~b} \sin \phi+k_{g} \mathrm{p} \cos \phi-k_{n} \mathrm{~b} \cos \phi+k_{n} \mathrm{p} \sin \phi=1 \\
& k_{g} \mathrm{q}-b^{\prime} \sin \phi-\phi^{\prime} \cos \phi \mathrm{b}-p^{\prime} \cos \phi+\phi^{\prime} \sin \phi \mathrm{p}-\tau_{g} \mathrm{~b} \cos \phi+\tau_{g} \mathrm{p} \sin \phi=0 \\
& k_{n} \mathrm{q}+b^{\prime} \cos \phi-\phi^{\prime} \sin \phi \mathrm{b}-p^{\prime} \sin \phi-\phi^{\prime} \cos \phi \mathrm{p}-\tau_{g} \mathrm{~b} \sin \phi-\tau_{g} \mathrm{p} \cos \phi=0 .
\end{aligned}
$$

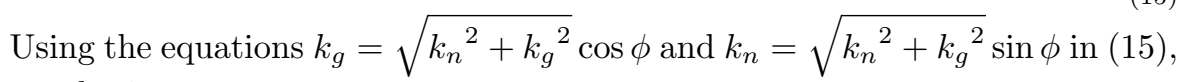
we obtain

$$
\begin{aligned}
\mathrm{q}^{\prime} & =1-\sqrt{{k_{n}}^{2}+{k_{g}}^{2}} \mathrm{p}, \\
\mathrm{p}^{\prime} & =\sqrt{{k_{n}}^{2}+k_{g}^{2}} \mathrm{q}-\left(\tau_{g}+\phi^{\prime}\right) \mathrm{b}, \\
\mathrm{b}^{\prime} & =\left(\tau_{g}+\phi^{\prime}\right) \mathrm{p} .
\end{aligned}
$$

If we differentiate (13) and use the formulas (16), we have

$$
\begin{aligned}
& \mathrm{rr}^{\prime}=\mathrm{q}-\mathrm{b}\left(\tau_{g}+\phi^{\prime}\right) \mathrm{p}, \\
& \mathrm{r} \mathrm{r}^{\prime}=\mathrm{q}-\mathrm{bb}^{\prime} .
\end{aligned}
$$

If the angular momentum vector, given in (9), is reconstructed by taking into account the position vector $\mathbf{x}$ aforementioned in (10), the angular momentum vector takes the form of

$$
\mathbf{H}_{O}=m v \mathrm{~b}(\cos \phi \mathbf{Y}+\sin \phi \mathbf{U})+m v \mathrm{p}(-\sin \phi \mathbf{Y}+\cos \phi \mathbf{U}) .
$$

Assume that

$$
\begin{aligned}
& h=\mathrm{p} v, \\
& w=\mathrm{b} v .
\end{aligned}
$$

By using the equation (12), let us express the vector $(\cos \phi \mathbf{Y}+\sin \phi \mathbf{U})$ in terms of $\mathbf{r}$ and $\mathbf{T}$ to determine the acceleration vector $\mathbf{a}$ in (8) along the tangent line $d$ and radial direction $B P$ in the plane $\Pi$. However, this is possible if and only if $\mathrm{p} \neq 0$. Since the component of the angular momentum along the normal of the plane $\Pi$ never vanishes according to the physical assumption, it can be immediately seen that $h \neq 0$ or $\mathrm{p} \neq 0$. Then we can write

$$
\cos \phi \mathbf{Y}+\sin \phi \mathbf{U}=\frac{1}{\mathrm{p}}(-\mathbf{r}+\mathrm{q} \mathbf{T}) .
$$


Also, when $\mathrm{p} \neq 0$, it is easy to get $\mathrm{r} \neq 0$ from (13). Hence, a unit vector $\mathbf{e}_{\mathbf{r}}$ can be defined as

$$
\mathbf{e}_{\mathbf{r}}=\frac{1}{\mathrm{r}} \mathbf{r}
$$

With the aid of (20) and (21), we have

$$
\cos \phi \mathbf{Y}+\sin \phi \mathbf{U}=\frac{1}{\mathrm{p}}\left(-\mathrm{r} \mathbf{e}_{\mathbf{r}}+\mathrm{q} \mathbf{T}\right) .
$$

If we substitute (22) in (8), we obtain the acceleration vector $\mathbf{a}$ as follows,

$$
\begin{aligned}
\mathbf{a} & =-\frac{\sqrt{k_{n}^{2}+k_{g}^{2}} v^{2} \mathrm{r}}{\mathrm{p}} \mathbf{e}_{\mathbf{r}}+\left(v \frac{d v}{d s}+\frac{\sqrt{k_{n}^{2}+k_{g}^{2}} v^{2} \mathrm{q}}{\mathrm{p}}\right) \mathbf{T} \\
& =S_{r} \mathbf{e}_{\mathbf{r}}+S_{t} \mathbf{T} .
\end{aligned}
$$

Here, $S_{t}$ and $S_{r}$ are the tangential and radial Siacci components of the acceleration, respectively. Using the equation $(19)_{1}$ with $\mathrm{p} \neq 0$, we get

$$
S_{r}=-\frac{\sqrt{k_{n}^{2}+k_{g}^{2}} \mathrm{r} h^{2}}{\mathrm{p}^{3}} .
$$

Now, let us investigate the different forms of the tangential component of acceleration, $S_{t}$ can be written in different forms. At first, if we consider the equations $(16)_{2,3}$ and $(19)_{1}$, we obtain

$$
\begin{aligned}
S_{t}=\frac{1}{2}\left(v^{2}\right)^{\prime}+\frac{\sqrt{k_{n}^{2}+k_{g}^{2}} v^{2} \mathrm{q}}{\mathrm{p}} & =\frac{\left(h^{2}\right)^{\prime}}{2 \mathrm{p}^{2}}+\frac{\left(\tau_{g}+\phi^{\prime}\right) \mathrm{b} h^{2}}{\mathrm{p}^{3}} \\
& =\frac{1}{2 \mathrm{p}^{2}}\left(\left(h^{2}\right)^{\prime}+\frac{h^{2}}{\mathrm{p}^{2}}\left(\mathrm{~b}^{2}\right)^{\prime}\right) .
\end{aligned}
$$

Similarly, using the equation $(17)_{1}$, we get

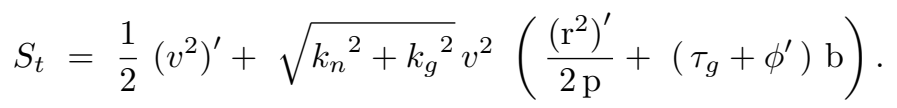

Finally, if we consider $(17)_{2}$ and also utilize (10) and (13), we obtain

$$
\begin{aligned}
S_{t} & =\frac{1}{2}\left(v^{2}\right)^{\prime}+\frac{\sqrt{k_{n}^{2}+k_{g}^{2}} v^{2}}{2 \mathrm{p}}\left(\mathrm{r}^{2}+\mathrm{b}^{2}\right)^{\prime} \\
& =\frac{1}{2}\left(v^{2}\right)^{\prime}+\frac{\sqrt{k_{n}^{2}+k_{g}^{2}} v^{2}}{2 \mathrm{p}}\langle\mathbf{x}, \mathbf{x}\rangle^{\prime}
\end{aligned}
$$

By considering the above derivation, we can express the following theorem:

Theorem 1 (Siacci's Theorem According to Darboux Frame). Let $P$ be a particle whose mass is $m$ and which moves on a regular surface $M$ in $E^{3}$ where the 
component of angular momentum along the unit vector $(-\sin \phi \mathbf{Y}+\cos \phi \mathbf{U})$ never vanishes. Then the acceleration vector a of $P$ can be stated as

$$
\mathbf{a}=-\frac{\sqrt{{k_{n}^{2}+k_{g}^{2}}^{2} v^{2} \mathrm{r}}}{\mathrm{p}} \mathbf{e}_{\mathbf{r}}+\left(v \frac{d v}{d s}+\frac{\sqrt{{k_{n}^{2}+k_{g}^{2}}^{2} v^{2}}}{\mathrm{p}}\right) \mathbf{T}=S_{r} \mathbf{e}_{\mathbf{r}}+S_{t} \mathbf{T} .
$$

$S_{t}$ lies along the tangent line of $C$, while $S_{r}$ is directed from $P$ towards the foot of the perpendicular that is from the origin of the space to the plane $\Pi$ to $C$ at $P$.

Example 1. Suppose that a particle $P$ moves on a right-handed circular helix lying on a cylinder which has a radius $A$. Let $(R, \theta, z)$ be a cylindrical coordinate system and according to this coordinate system, let the position vector of the particle $P$ be given as

$$
\mathbf{x}=A \mathbf{e}_{R}+z \mathbf{k}, \quad z=B \theta
$$

Here, $\mathbf{e}_{R}=\cos \theta \mathbf{i}+\sin \theta \mathbf{j}, \quad \mathbf{e}_{\theta}=-\sin \theta \mathbf{i}+\cos \theta \mathbf{j}$, where $\mathbf{i}, \mathbf{j}$, and $\mathbf{k}$ compose a right-handed orthonormal frame and $A, B$ are positive constants (Fig.2). Also, let $\mathbf{k}$ be the axis of the helix and $\alpha$ be the helix angle where $\tan \alpha=A / B$. Let $\dot{\theta}$ be denoted by $\omega$. Then the velocity vector and the acceleration vector of $P$ can be obtained as

$$
\mathbf{v}=\omega\left(A \mathbf{e}_{\theta}+B \mathbf{k}\right), \quad \mathbf{a}=-A \omega^{2} \mathbf{e}_{R}+\dot{\omega}\left(A \mathbf{e}_{\theta}+B \mathbf{k}\right)
$$

Let $\mathrm{C}=\sqrt{A^{2}+B^{2}}$. Thus, the speed of $P$ and its time derivative can be obtained as

$$
v=\dot{s}=\mathrm{C} \omega, v \frac{d v}{d s}=\mathrm{C} \dot{\omega} .
$$

From $(30)_{1}$, it can be easily seen that the arc length increases linearly with $\theta$. Considering $\tan \alpha=A / B$, where $A>0$ and $B>0$, one can easily find the Frenet-Serret vectors as

$$
\mathbf{T}=\sin \alpha \mathbf{e}_{\theta}+\cos \alpha \mathbf{k}, \mathbf{N}=-\mathbf{e}_{R}, \mathbf{B}=-\cos \alpha \mathbf{e}_{\theta}+\sin \alpha \mathbf{k} .
$$

If we use the relation matrix between the Frenet frame and the Darboux frame, the Darboux basis of the helix is given by

$$
\begin{aligned}
& \mathbf{T}=\sin \alpha \mathbf{e}_{\theta}+\cos \alpha \mathbf{k} \\
& \mathbf{Y}=-\cos \phi \mathbf{e}_{R}+\sin \phi \cos \alpha \mathbf{e}_{\theta}-\sin \phi \sin \alpha \mathbf{k} \\
& \mathbf{U}=-\sin \phi \mathbf{e}_{R}-\cos \phi \cos \alpha \mathbf{e}_{\theta}+\cos \phi \sin \alpha \mathbf{k}
\end{aligned}
$$




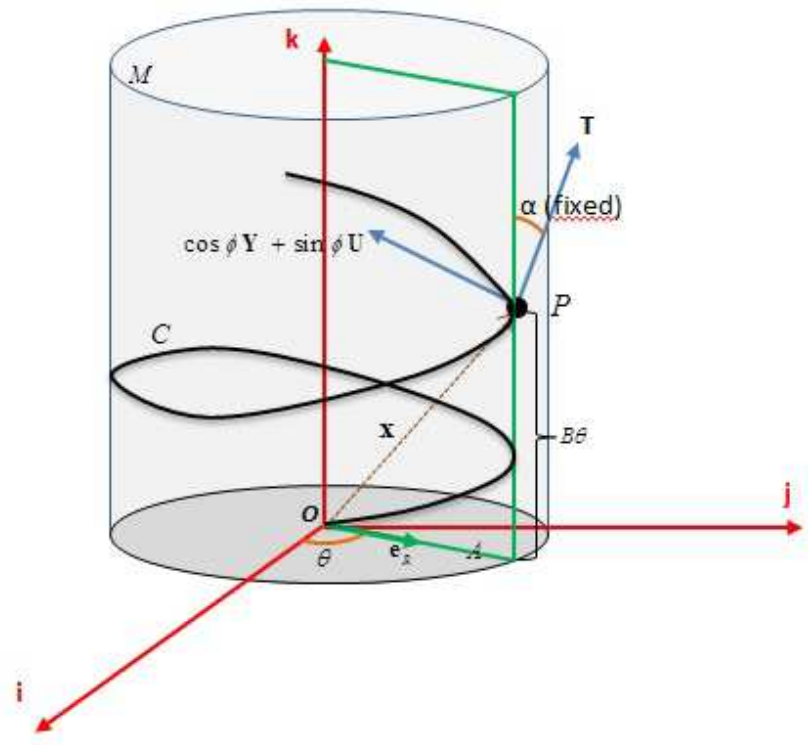

Figure 2: A particle $P$ moves on a right-handed circular helix lying on a cylinder which has a radius $A$. The position vector of $P$ at time $t$ is $\mathbf{x}=$ $A \mathbf{e}_{R}+B \theta \mathbf{k}$. Also, $\mathbf{k}$ is the axis of the helix and $\alpha$ is the helix angle. 
The curvature $\kappa$ and the torsion $\tau$ are constants: $\kappa=\frac{A}{\mathrm{C}^{2}}, \tau=\frac{B}{\mathrm{C}^{2}}$. Thus, from (4), the geodesic torsion, geodesic curvature, and normal curvature can be found as follows,

$$
\begin{aligned}
& \tau_{g}=\frac{B}{\mathrm{C}^{2}}-\phi^{\prime}, \\
& k_{g}=\frac{A}{\mathrm{C}^{2}} \cos \phi, \\
& k_{n}=\frac{A}{\mathrm{C}^{2}} \sin \phi .
\end{aligned}
$$

From (11), (28) 1 , and (32), we obtain

$$
\begin{aligned}
& \mathrm{q}=z \cos \alpha, \\
& \mathrm{p}=A, \\
& \mathrm{~b}=z \sin \alpha .
\end{aligned}
$$

If we substitute (34) in (10), after some calculations, we have

$$
\mathbf{x}=z \cos \alpha \mathbf{T}-(z \sin \alpha \sin \phi+A \cos \phi) \mathbf{Y}+(z \sin \alpha \cos \phi-A \sin \phi) \mathbf{U} .
$$

From (35), it is possible to see the Darboux components of $\mathbf{x}$.

Using $(19)_{1,2}$ and $(34)_{2,3}$, the components for the angular momentum per unit mass can be obtained as

$$
h=A \mathrm{C} \omega \quad, \quad w=A z \omega .
$$

If we give the Siacci's theorem according to Darboux frame, we find that

$$
S_{r}=-\mathrm{r} \omega^{2}=-\omega^{2} \sqrt{A^{2}+z^{2} \cos ^{2} \alpha} \quad, \quad S_{t}=\mathrm{C} \dot{\omega}+\omega^{2} z \cos \alpha .
$$

\section{References}

[1] F. Siacci, Moto per una linea gobba. Atti R Accad Sci. Torino 14 (1879), 946-951.

[2] B. O’NeIL, Elemantary Differential Geometry. Academic Press, Newyork, 1966.

[3] S. P. Radzevich, Geometry of Surfaces: A practical Guide for Mechanical Engineers. Wiley, 2013.

[4] J. CASEy, Siacci's Resolution of the Acceleration Vector for a Space Curve. Meccanica 46 (2011), 471-476 DOI:10.1007/s11012-010-9296-x.

[5] E. T. Whittaker, A Treatise on the Analytical Dynamics of Particles and Rigid Bodies. 4th Edition, Cambridge University Press, 1944.

[6] N. Grossman, The Sheer Joy of Celestial Mechanics. Birkhauser, Basel, 1996. 
[7] M.Y. Yilmaz, M. Bektaş and Z. KüÇüKarslan, Siacci's Theorem for Curves in Finsler Manifold $\mathbf{F}^{\mathbf{3}}$. Turkish Journal of Science and Technology 7(2) (2012), 181-185.

[8] T. Shifrin, Differential Geometry: A First Course in Curves and Surfaces. University of Georgia, Preliminary Version, 2008.

[9] F. DoG̃an and Y. Yayli, Tubes with Darboux Frame. Int. J. Contemp. Math. Sciences 7(16) (2012), 751-758.

[10] F. DoG̃an, Generalized Canal Surfaces. Ankara University, Graduate School of Natural and Applied Sciences, Ankara, Ph.D. Thesis, 2012.

[11] Ö. BeKtaş And S. YüCE, Special Smarandache Curves According to Darboux Frame in $E^{3}$. Romanian Journal of Mathematics and Computer Science 3(1) (2013), 48-59.

Kahraman Esen Ozen

Faculty of Arts and Sciences, Sakarya University,

54187 Sakarya, Turkey,

E-mail: kahraman.ozen1@ogr.sakarya.edu.tr

Murat Tosun

Department of Mathematics,

Faculty of Arts and Sciences, Sakarya University,

54187 Sakarya, Turkey,

E-mail: tosun@sakarya.edu.tr

Mahmut Akyiğit

Department of Mathematics,

Faculty of Arts and Sciences, Sakarya University,

54187 Sakarya, Turkey,

E-mail: makyigit@sakarya.edu.tr 